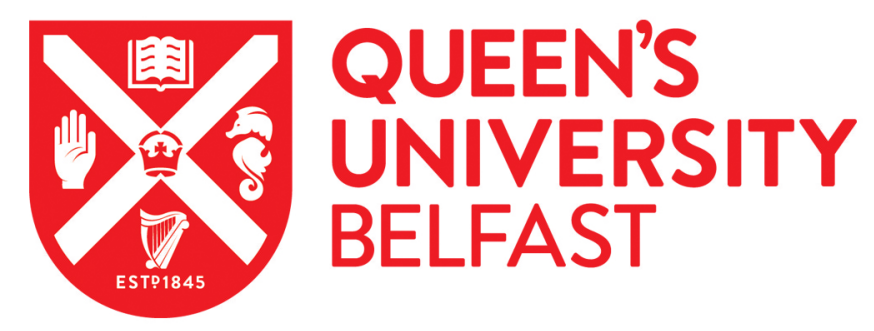

\title{
Underwater surveying and mapping using rotational potential fields for multiple autonomous vehicles
}

\author{
McIntyre, D., Naeem, W., McIntyre, D., Ali, S. S. A., \& Anwar, A. (2017). Underwater surveying and mapping \\ using rotational potential fields for multiple autonomous vehicles. In 2016 IEEE International Conference on \\ Underwater System Technology: Theory and Applications (USYS) (pp. 77-82) \\ https://doi.org/10.1109/USYS.2016.7893926
}

Published in: 2016 IEEE International Conference on Underwater System Technology: Theory and Applications (USYS)

\section{Document Version:}

Peer reviewed version

Queen's University Belfast - Research Portal:

Link to publication record in Queen's University Belfast Research Portal

\section{Publisher rights}

(C) 2017 IEEE. This is an open access article published under a Creative Commons Attribution License

(https://creativecommons.org/licenses/by/4.0/), which permits unrestricted use, distribution and reproduction in any medium, provided the author and source are cited.

\section{General rights}

Copyright for the publications made accessible via the Queen's University Belfast Research Portal is retained by the author(s) and / or other copyright owners and it is a condition of accessing these publications that users recognise and abide by the legal requirements associated with these rights.

Take down policy

The Research Portal is Queen's institutional repository that provides access to Queen's research output. Every effort has been made to ensure that content in the Research Portal does not infringe any person's rights, or applicable UK laws. If you discover content in the Research Portal that you believe breaches copyright or violates any law, please contact openaccess@qub.ac.uk. 


\section{Underwater Surveying and Mapping using Rotational Potential Fields for Multiple Autonomous Vehicles}

\author{
David McIntyre \\ School of Electronics, Electrical \\ Engineering and Computer Science Engineering and Computer Science \\ Queen's University Belfast \\ Email: dmcintyre04@qub.ac.uk \\ Wasif Naeem \\ School of Electronics, Electrical \\ Queen's University Belfast \\ Email:w.naeem@qub.ac.uk
}

\author{
Syed Saad Azhar Ali and Atif Anwer \\ Center for Intelligent Signal \& Imaging Research, \\ Universiti Teknologi PETRONAS, \\ 32610 Seri Iskandar, Perak, Malaysia \\ Email: saad.azhar@utp.edu.my
}

\begin{abstract}
This paper presents a new technique for exploration and mapping/surveying of underwater infrastructure and/or objects of interest, using multiple autonomous underwater vehicles (AUVs). The proposed method employs rotational potential fields, and extends them for use on multiple vehicles within a three dimensional environment. An inter-vehicle fluid formation is maintained throughout, free of angular constraints (or the need of a virtual vehicle). When an object of interest is approached, the formation is split and follows a smooth trajectory around opposite sides of its boundary. To fully utilise the potential of rotational fields, a unique local 2D-plane is created around every object within the 3D environment, which is employed for boundary coverage. Traditional artificial potential fields are used to guide vehicles towards each object in turn (and maintain the fluid formation), while rotational fields are employed within the local 2D-plane providing a smooth trajectory around opposing sides of every object. Simulation results show the method to be effective, providing a more stable trajectory. Comparison with the standard technique shows that the formation is maintained throughout and overall journey time is significantly reduced using this method.

Keywords - rotational potential fields, artificial potential fields, obstacle avoidance, cooperative control, mapping and surveying, autonomous underwater vehicles
\end{abstract}

\section{INTRODUCTION}

The first real developments in the field of autonomous underwater vehicles (AUVs) began in the 1980s, following the increase of low-powered computers coupled with improved software capabilities. Research funding increased during the 1990 s, and the advent of the 21 st century heralded a new age of underwater exploration, when the commercial market began to grow. AUVs range from huge, multi-million dollar behemoths weighing over 60 tonnes (used for large scale industrial projects) [1], right down to small, hand-held models designed for hobbyists with a price tag of just a few hundred dollars [2]. Uses are widespread, covering different fields of interest, for example, scientific applications include water-testing, datagathering, off-shore mapping, marine biology inspection etc. [3], industrial and commercial applications (dominated by the oil and gas industry [4]) include the inspection of underwater structures such as oil rigs and pipelines [5], and the military use them for intelligence-gathering, communications, navigation and eradicating underwater mines [6] to name a few.
Recently there has been an increase in popularity for small, inexpensive models, and concerns are being raised as to the impact on the underwater environment [7].

Over the past number of years, the great need for multiple AUVs (MAUVs) working together within a cooperative framework has been highlighted following crises such as the BP oil spill disaster in 2010 [8] and the tragic disappearance (and subsequently, so far unsuccessful search) of Malaysia Airlines flight MH370 in 2014 [9]. This is now a very viable prospect given today's technology, along with developments in communications, and research is growing in this area. The advantages of using a fleet of vehicles over a single entity are many and varied, for example, in the case of mapping and/or exploration, far greater area-coverage can be achieved in a reduced time-frame. Further, the inclusion of heterogeneous vehicles within a fleet can greatly expand the range of mission objectives, with a variety of tasks being performed in a single visit [10]. Groups could vary in size also from large scale search-and-rescue teams covering vast swathes of the ocean to small, compact formations armed with a specific task e.g. pipeline inspection [11].

The cooperative control framework around multiple vehicles contains a number of prominent challenges including collision avoidance (both intra and inter-vehicle), localisation and communications. As a vehicle's depth increases it is forced to rely on dead-reckoning techniques (or periodic resurfacing) to keep accurate track of its position, as GPS does not exist underwater. Underwater communication is limited and severely attenuated by salinity levels and low bandwidth, these, coupled with multi-path echoing pose challenges to real-time reception [12]. Power and energy consumption during longterm missions, alongside operation within the confines of a dynamic and uncertain environment, can lead to vehicle failure or loss. Collision avoidance is also a major area, and essential for improved autonomy and mission success. Challenges within this field include inter-vehicle collision in group formations alongside both static and dynamic obstacles. Path-planning and guidance are additional spheres within which solid design consideration is a necessity. Thus, to enable true cooperation 
between platforms, proper design of control architecture is essential.

\section{BACKGROUND AND OVERVIEW}

To overcome the challenges listed above, a number of techniques and control architectures have been developed in recent years. These can be broadly classified into two main categories - centralised and decentralised. In the centralised architecture, each vehicle is linked to a central controller and periodically receives information pertaining to the current mission, whereas in the decentralised architecture, independent agents generally act alone, whilst maintaining communication links to some or all of the other agents. The two categories are not mutually exclusive and may include aspects of either area, depending on a range of factors such as fleet size, sensor range and mission objectives to name a few. The above techniques are further classified into three broad fields - behaviour-based, leaderfollower and virtual structures (falling into both centralised and decentralised domains and/or a mix of the two).

Behaviour-based: inspired by the animal world, vehicles operating under this model generally perform sense-and-react manoeuvring. Behavioural rules vary dramatically in both scope and speed and depend upon a range of variables linked to mission objectives, working environment, vehicle capabilities etc. and rule prioritisation may be organised by the assignment of iterative cost functions. Successful use of the behaviour-based strategy has been made in the modelling of fish schooling behaviour [13].

Leader-follower: a single vehicle (or vehicles), programmed with path-planning or guidance capabilities leads a group of follower agents towards a point of interest. Cost reduction can be considerable as followers are generally only concerned with their position relative to the leader, requiring less complex hardware. A leader vehicle may transmit concurrent coordinates to each follower or remain independent, and certain followers can be suitably equipped to replace leader vehicles for system robustness in the case of leader failure [14].

Virtual Structures: MAUVs often group into a rigid formation or shape which then moves in its entirety through an environment towards a goal position. An imaginary vehicle may be employed during this technique, placed at a strategic position within the group, enabling all others to maintain a specified distance and angle from it to preserve the formation [15].

\section{A. Artificial Potential Fields}

In the $1980 \mathrm{~s}$, research by $\mathrm{O}$. Khatib guided a vehicle to an intended goal, whilst avoiding obstacles, using the analogy of a potential field. A combination of repulsive fields surrounding obstacles, coupled with an attraction at the intended goal position, creating an overall field, was used to guide the agent [16]. An attractive field towards the goal position was created using Equation 1

$$
U x_{d}(x)=\frac{1}{2} k\left(x-x_{d}\right)^{2}
$$

where $U x_{d}(x)$ is the attractive potential field at the goal, $x$ is the current position of the agent, $x_{d}$ is desired goal and $k$ is a scaling factor.

To ensure a collision-free journey, repulsive fields were formed around obstacles to prevent collisions using Equation 2.

$$
U_{o}(x)=\left\{\begin{aligned}
\frac{1}{2} \eta\left(\frac{1}{\rho}-\frac{1}{\rho_{o}}\right)^{2} & \text { if } \rho \leq \rho_{o} \\
0 & \text { if } \rho \geq \rho_{o}
\end{aligned}\right.
$$

where $U_{o}(x)$ is the repulsive force around each obstacle, $\rho_{o}$ is the limit distance of the potential field, $\rho$ is the shortest distance from vehicle to obstacle $O$ whilst $\eta$ is a scaling factor.

The popularity and use of APFs has fluctuated over the decades but, being computationally inexpensive, they have been used in a number of different areas and also enhanced to include multiple vehicles [17]. Some papers have extended their use into three-dimensional work for both airborne [18], and underwater vehicles [19]. A frequently reported issue with this method is when attractive and repulsive forces combine to produce a null force, leading the vehicle to become trapped in a local minimum and remain in a static position. A further issue prevents a goal position to be achieved due to a nearby obstacle and is commonly known as the GNRON problem (goal nonreachable by obstacles nearby). Various solutions to solve these problems have been proposed, such as simulated annealing [20].

\section{B. Rotational Potential Fields}

Rotational potential fields (RPFs), a variant of APFs, have also been employed to solve the local minima problem by driving vehicles around obstacles in a circular manner. Clockwise or anticlockwise fields are created using tangential vectors in place of direct repulsion (see Fig 1), the force of which increases as vehicle distance-to-obstacle decreases.
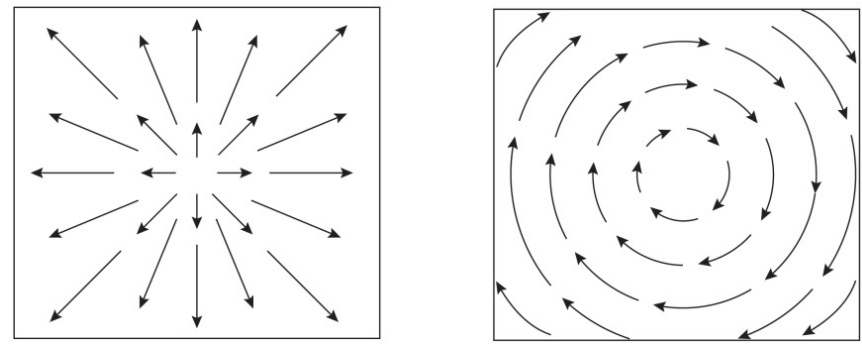

Fig. 1. Original Potential Fields vs Rotational Potential Fields [21]

RPFs, by their very nature, work most efficiently within a two dimensional plane, and research has mainly been focused in this area. For example, in [22] a single ground-based, wheeled robot is guided towards a goal within a 2D environment, whilst RPFs guide it around obstacles on a nearest-edge basis. Work involving multiple vehicles also tends to focus on the guidance of rigid vehicle structures. For instance, in [23] the entire vehicle formation is guided towards a goal without splitting (on a 2D plane), using RPFs for obstacle avoidance. In [24], use is made of a virtual vehicle to guide a rigid 
2D group of vehicles to an intended target, which meets an obstacle head on before splitting and recombining. Extending the technique into three dimensions, in [25] a single unmanned aircraft uses rotational fields to avoid obstacles, again on a nearest-edge basis.

This paper presents a novel technique allowing rotational fields to be employed within a three dimensional environment, primarily for surveying and mapping applications. A pair of vehicles will be guided in a fluid formation, using traditional APFs, towards objects of interest, before encircling them on both sides of their boundaries, using RPFs with a smooth trajectory. Rotational fields significantly reduce the issue of local minima and adjustable waypoints (to increase or decrease the diameter of the circular field) tackle the GNRON problem. In addition, a rigid structure will be dispensed with in favour of a fluid formation, eliminating the need for a virtual vehicle thus reducing computational load. Vehicles will employ both attractive and repulsive fields to maintain a user-defined separation without angular constraints. Every object of interest in turn will be surveyed within a $3 \mathrm{D}$ environment and the technique could be exercised for a number of useful applications, for example, in the area of mapping and exploration.

The remainder of the paper is organised as follows. Section III explains the proposed methodology, covering path-planning and potential fields. In Section IV the fluid formation and object survey are detailed, along with the formation of the 2D plane and translation between $\mathbb{R}^{2} \& \mathbb{R}^{3}$. Section $\mathrm{V}$ displays the simulation results and their analysis, with the conclusion following in Section VI.

\section{Proposed Methodology}

\section{A. Path Planning}

Initially a pair of vehicles, $v_{1} \& v_{2}$, are placed within a three-dimensional Euclidean space $\mathbb{R}^{3}$ populated by a set of objects $O$. To guide vehicles towards objects of interest in turn, a number of waypoints $w$ are determined, to act as attractive poles for use with traditional potential fields in a method similar to that used by the authors in [26]. Waypoints must be positioned in order to appropriately guide $v_{1} \& v_{2}$ towards the centre of each object $O$ in turn. Initially, the midpoint $m$ is used between the starting positions of $v_{1} \& v_{2}$, an imaginary line is then drawn between $m$ and the centre point of the first object $O_{1}$. The initial waypoint $w_{1}$ is then placed on the opposite side of $O_{1}$ at a user-defined distance dependent on $O_{1}$ 's radius. $m$ is then replaced by $w_{1}$ and the process is repeated for $w_{2}$ which is placed beyond $\mathrm{O}_{2}$. This is repeated until every object has a corresponding waypoint.

\section{B. Potential Fields}

Following the determination of the waypoints, both vehicles are guided by traditional APFs (see Section II) towards each object of interest in turn. As vehicles approach the object boundary they are split by rotational fields, which guide them around opposing sides, towards the current waypoint.

Traditional Fields: Potential fields work use a gradient descent method i.e. an attractive pole acts in a similar manner to an energy well, driving vehicles towards its lowest point. Repulsive fields can be imagined as 'rounded hills'protruding from this energy well, for the purpose of obstacle avoidance. At each coordinate within the working environment, the negative gradient can be determined and there are many variations on the original equations. Attractive and repulsive vectors are then summed at each point to produce an overall force vector. Attractive goals or waypoints can be described by using the gradient equation (3)

$$
\mathbf{F}_{w_{i}}^{a t t}(\mathbf{x})=-\nabla U_{w_{i}}^{a t t}(\mathbf{x})=k_{a}\left(\mathbf{x}-\mathbf{x}_{w_{i}}\right)
$$

where $\mathbf{F}_{w_{i}}^{a t t}(\mathbf{x})$ is the attractive force vector due to waypoint $i$ acting on the coordinates $\mathbf{x}$ in a three-dimensional Euclidean space $\mathbb{R}^{3}$ (herein after referred to as a point or position), $-\nabla U_{w_{i}}^{a t t}(\mathbf{x})$ is the negative gradient due to the attractive force of waypoint $i$ at the point $\mathbf{x}, k_{a}$ is a force scaling factor and $\mathbf{x}_{w_{i}}$ is the position of waypoint $i$.

Repulsion, radiating isotropically from a point, can be described by (4), where the force is a factor of the squared distance between the vehicle and repulsive centre.

$$
\mathbf{F}_{o_{i}}^{r e p}(\mathbf{x})=-\nabla U_{o_{i}}^{r e p}(\mathbf{x})=k_{r}\left(\mathbf{x}-\mathbf{x}_{o_{i}}\right) d^{-3 / 2}
$$

where $\mathbf{F}_{o_{i}}^{r e p}(\mathbf{x})$ is the repulsive force vector due to object $i$ acting at the point $\mathbf{x},-\nabla U_{o_{i}}^{r e p}(\mathbf{x})$ is the negative gradient due to the repulsive force of object $i$ at the point $\mathbf{x}, k_{r}$ is a force scaling factor, $\mathbf{x}_{o_{i}}$ is the position of object $i$ and $d$ is the shortest distance between the vehicle and object.

A number of points are used within $\mathbb{R}^{3}$ to approximate the shape of an object's surface. Each of these acts as a point-source repulsion, and the combined sum of vector forces serves as an overall repulsion at each coordinate within $\mathbb{R}^{3}$. However, it should be noted that this combined force is only employed within a distance threshold of the object surface, and is described by (5)

$$
\begin{aligned}
& \mathbf{F}_{o_{\text {res }}}^{r e p}(\mathbf{x})=-\nabla U_{o_{\text {res }}}^{r e p}(\mathbf{x})= \\
& \left\{\begin{aligned}
\sum_{p_{o}=1}^{p_{o}=m} k_{r}\left(\mathbf{x}_{N_{i}}-\mathbf{x}_{o_{i}}\right) d_{p_{o}}^{-3 / 2} & \text { if } d_{p_{o}} \leq r_{o_{i}} \\
0 & \text { if } d_{p_{o}}>r_{o_{i}}
\end{aligned}\right.
\end{aligned}
$$

where $\mathbf{F}_{\text {ores }_{\text {res }}}^{\text {rep }}(\mathbf{x})$ is the overall resultant force vector acting on a point $\mathbf{x}$ due to the sum of every repulsive vector emanating from a set of $m$ points on the surface of an object $O,-\nabla U_{o_{\text {res }}}^{r e p}(\mathbf{x})$ represents the negative gradient due to this resultant force, $k_{r}$ is a force scaling factor, $\mathbf{x}_{N_{i}}$ is the position of vehicle $i \in N$ (where $N$ is the set of vehicles), $\mathbf{x}_{o_{i}}$ is a point on the surface of the object, $d_{p_{o}}$ is the distance from the vehicle to the current object surface point and $r_{o_{i}}$ is the range of influence of object repulsion.

Rotational Fields: In order to achieve clockwise and anticlockwise fields around each object, tangential force vectors were formed on the $x-y, \mathbb{R}^{2}$ plane. In order to accomplish this, each resultant force vector radiating isotropically from an object $\left(\mathbf{F}_{\text {ores }_{\text {rep }}}^{\text {rep }}\right.$ - determined from Equation 5), is broken down into its $x, y \& z$ component vectors. As opposing rotational fields are only achieved on a 2D plane, the $z$ component is 
removed, leaving the $x \& y$ vectors. These two vectors are then either summed of subtracted in order to create the rotational fields around the object.

\section{Fluid Formation And ObJect SuRvey}

\section{A. Fluid Formation}

Here, a novel approach is used which dispenses with the need for a rigid structure and/or a virtual vehicle. The lack of angular constraint produces a more fluid flow, yet ensures vehicles maintain an approximate separation distance $s_{v}$, when not in surveying mode. Depending on the distance between $v_{1} \& v_{2}$, APF Equations $3 \& 4$ are used to assign either an attractive or repulsive force to $v_{2}$, which then acts upon $v_{1}$ depending on the desired separation, $d_{m i n}$, set by the user.

\section{B. Object Survey}

As the vehicle pair approaches each object, $v_{1} \& v_{2}$ must each be assigned an opposing rotational field to ensure that the entire item of interest is encircled. The process of determining which vehicle is assigned either a clockwise or anti-clockwise field involves the use of three vectors. Firstly, as a reference, the vector $\overrightarrow{O_{i} w_{i}}$ between the current object centre and its corresponding waypoint is calculated. Further vectors are evaluated from both $v_{1} \& v_{2}$ to $O_{i}, \overrightarrow{v_{1} O_{i}} \& \overrightarrow{v_{2} O_{i}}$. The anticlockwise angle is then measured from $\overrightarrow{O_{i} w_{i}}$ to both $\overrightarrow{v_{1} O_{i}}$ \& $\overrightarrow{v_{2} O_{i}}$ to produce $\alpha \& \theta$ respectively. At a predefined distance from the object boundary a comparison is made between $\alpha$ $\& \theta$. If $\alpha \geq \theta, v_{1}$ is assigned a clockwise field, else anitclockwise. To ensure complete boundary coverage $v_{2}$ uses the opposite rotational field to $v_{1}$ in all instances.

\section{Translating Between $\mathbb{R}^{3}$ and $\mathbb{R}^{2}$}

The use of rotational fields in the $x-y$ plane disrupts the smooth trajectory of a vehicle within $\mathbb{R}^{3}$, as seen in Fig 2 .

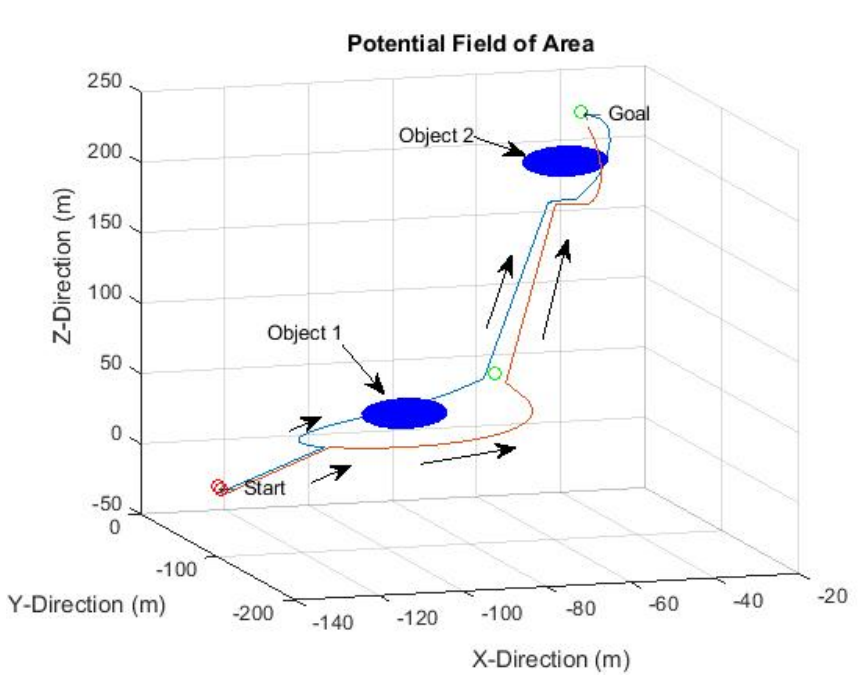

Fig. 2. Example of $x-y$ rotational fields within $\mathbb{R}^{3}$

Fig 2 above shows a vehicle pair encircling two objects before arriving at a goal position. In the case of object 1 , a near-horizontal approach shows a relatively smooth trajectory around the object's boundary. The approach towards object 2 , however, takes a much steeper angle: it can be seen that the trajectory of both vehicles around the object is impaired by the horizontal repulsion. This technique therefore creates a unique local 2D plane around each object within which a smooth rotation can be achieved. The local $2 \mathrm{D}$ plane in question is determined when both vehicles enter the range of influence of the rotational fields, and it lies on the points connecting the object centre with $v_{1} \& v_{2}$. Creating a two dimensional plane within a $3 \mathrm{D}$ environment, whereby coordinates can be easily interchanged between the two, involves a number of steps. To illustrate this, the case of two vehicles $\left(v_{1} \& v_{2}\right)$ and an object $\left(O_{i}\right)$ within $\mathbb{R}^{3}$ will be used. The $2 \mathrm{D}$ plane will be formed such that $v_{1}, v_{2}$ and $O_{i}$ all lie upon it simultaneously.

The steps involve creating a transformation matrix which will enable coordinates to be converted from $\mathbb{R}^{3}$ (the global reference frame) onto the $\mathbb{R}^{2}$ plane (local reference frame). Let both vehicles have coordinates $v_{1}=\left(v_{1_{x}}, v_{1_{y}}, v_{1_{z}}\right) \& v_{2}=$ $\left(v_{2_{x}}, v_{2_{y}}, v_{2_{z}}\right)$ and object $O_{i}=\left(O_{i_{x}}, O_{i_{y}}, O_{i_{z}}\right)$.

1) Creating Local Reference Frame: Step 1: move object to the centre of the new origin i.e.

$O_{i}^{\prime}=\left(0-O_{i_{x}}, 0-O_{i_{y}}, 0-O_{i_{z}}\right)=\left(-O_{i_{x}},-O_{i_{y}},-O_{i_{z}}\right)$

Step 2: apply the same shift to $v_{1} \& v_{2}$ i.e. $v_{1}^{\prime}=\left(v_{1_{x}}-\right.$ $\left.O_{i_{x}}, v_{1_{y}}-O_{i_{y}}, v_{1_{z}}-O_{i_{z}}\right) \& v_{2}^{\prime}=\left(v_{2_{x}}-O_{i_{x}}, v_{2_{y}}-O_{i_{y}}, v_{2_{z}}-\right.$ $\left.O_{i_{z}}\right)$

To successfully translate between reference frames, three new axes within the local environment are required to represent the translated $x, y \& z$ coordinates. These are assigned by the formation of three unit vectors (using both vehicle and objects from the global frame) as in step 3 below.

Step 3: the first unit vector is formed by the normalisation of the vector between the $O_{i}$ and $v_{1}$, and acts as the $x$-axis of the new local frame.

$$
\hat{\mathbf{t}}=\frac{\left(v_{1}^{\prime}-O_{i}^{\prime}\right)}{\left|\left(v_{1}^{\prime}-O_{i}^{\prime}\right)\right|}
$$

Step 4: taking the normalisation of the cross product of $\hat{\mathbf{t}}$ and the vector between $O_{i} \& v_{2}$ gives a vector orthogonal to $\hat{\mathbf{t}}$ which acts as the $y$-axis of the new local frame.

$$
\hat{\mathbf{n}}=\frac{\hat{\mathbf{t}} \times\left(v_{1}^{\prime}-O_{i}^{\prime}\right)}{\left|\left(\hat{\mathbf{t}} \times\left(v_{1}^{\prime}-O_{i}^{\prime}\right)\right)\right|}
$$

Step 5: the local frame $z$-axis is formed from the unit vector of the cross product between $\hat{\mathbf{t}}$ and $\hat{\mathbf{n}}$.

$$
\hat{\mathbf{b}}=\frac{(\hat{\mathbf{t}} \times \hat{\mathbf{n}})}{|(\hat{\mathbf{t}} \times \hat{\mathbf{n}})|}
$$

2) Transformation Matrices: To translate coordinates from the $\mathbb{R}^{3}$ onto the $\mathbb{R}^{2}$ plane, a transformation matrix can be determined, such that multiplying $\mathbb{R}^{3}$ coordinates with the matrix produces the new coordinates on $\mathbb{R}^{2}$.

Step 6: translation to the new $x, y \& z$-axes is performed using the unit vectors, which are combined to produce the new 
Global $\rightarrow$ Local transformation matrix, as given by Equation 9.

$$
\mathbf{G}=\left[\begin{array}{lll}
\hat{t}_{x} & \hat{b}_{x} & \hat{n}_{x} \\
\hat{t}_{y} & \hat{b}_{y} & \hat{n}_{y} \\
\hat{t}_{z} & \hat{b}_{z} & \hat{n}_{z}
\end{array}\right]
$$

Step 7: let $\mathbf{P}$ denote the current position of a vehicle in $\mathbb{R}^{3}$. Before translation, the position of object $O_{i}$ must be subtracted from $\mathbf{P}$ : $\mathbf{P s}=\mathbf{P}$ - $\mathbf{O b}$.

Step 8: $\mathbf{G}$ is now used to translate coordinates from the global to the local plane.

$$
\left[P s^{\prime}\right]=[G][P s]
$$

Step 9: the vehicles position is converted back from $\mathbb{R}^{2}$ into $\mathbb{R}^{3}$ using the transpose of $\mathbf{G}$.

$$
[P s]=\left[G^{T}\right]\left[P s^{\prime}\right]
$$

Step 10: finally the object coordinates $\mathbf{O b}$ are added to Ps to give its true position within $\mathbb{R}^{3}$.

\section{Simulation Results}

The MATLAB software package was used to test the validity of the proposed approach, and compare it against a similar method employing a purely horizontal repulsive force surrounding each object. A scenario was utilised within which a pair of vehicles were given the task of surveying five objects (around opposite sides of their boundaries) in a 3D environment. To fully evaluate the method, the objects are such arranged that the vertical ascent of both vehicles is increased throughout their journey. It is assumed that each vehicle has omnidirectional movement with six degrees of freedom and a negligible turning radius. Vehicles operate at a constant velocity within a disturbance free environment, thus the only threats are either object or inter-vehicle collisions.

Scenario: vehicles $v_{1} \& v_{2}$ have starting coordinates (-140,$100,-5)$ and $(-140,-105,-5)$ respectively with an inter-vehicle separation distance set at $3 \mathrm{~m}$. Five objects of radius $10 \mathrm{~m}$ are each placed at $(-90,-60,5),(-15,0,30),(0,0,110),(5,5,190)$ and $(7.5,7,5.300)$, intermediate waypoints are placed at a distance of $25 \mathrm{~m}$ from an object's surface (employing a circle of acceptance of $1.5 \mathrm{~m}$ ) and the range of object repulsion is determined as $20 \mathrm{~m}$. Force scale factors are shown in Table I.

TABLE I

SCALE FACTOR QUANTITIES FOR POTENTIAL FIELD EQUATIONS

\begin{tabular}{|l|l|l|l|}
\hline \multicolumn{4}{|c|}{ Force Scale Factors for Potential Fields } \\
\hline $\begin{array}{l}\text { Attractive Goal } \\
\text { or Waypoint }\end{array}$ & $\begin{array}{l}\text { Object } \\
\text { Repulsion }\end{array}$ & $\begin{array}{l}\text { Inter-vehicle } \\
\text { Attraction }\end{array}$ & $\begin{array}{l}\text { Inter-vehicle } \\
\text { Repulsion }\end{array}$ \\
\hline 0.65 & $1 \times 10^{4}$ & $1 \times 10^{-} 5$ & 3 \\
\hline
\end{tabular}

Results: the scenario described above was performed using both horizontal repulsion for the rotational fields, followed by the proposed technique involving 2D sub-planes, the results are shown below in Figs $3 \& 4$ respectively.

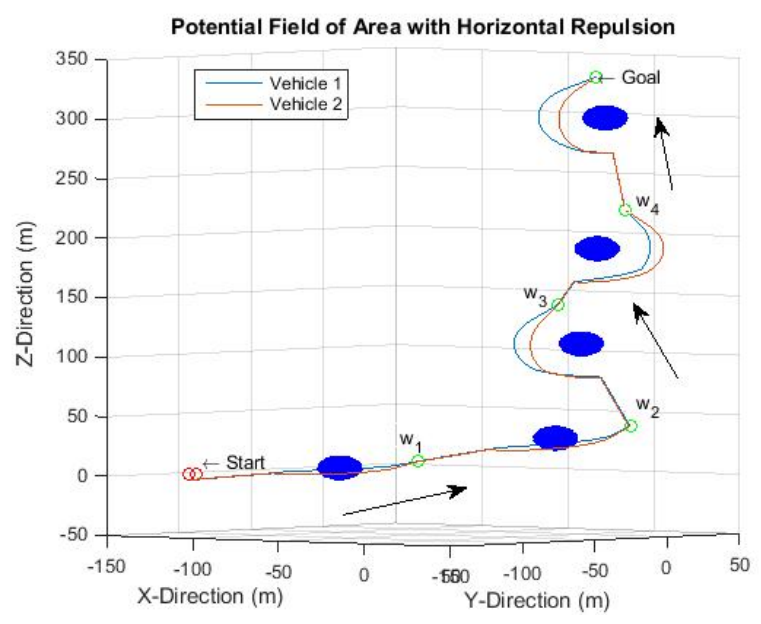

Fig. 3. The Survey of Five Objects using Horizontal Rotational Fields

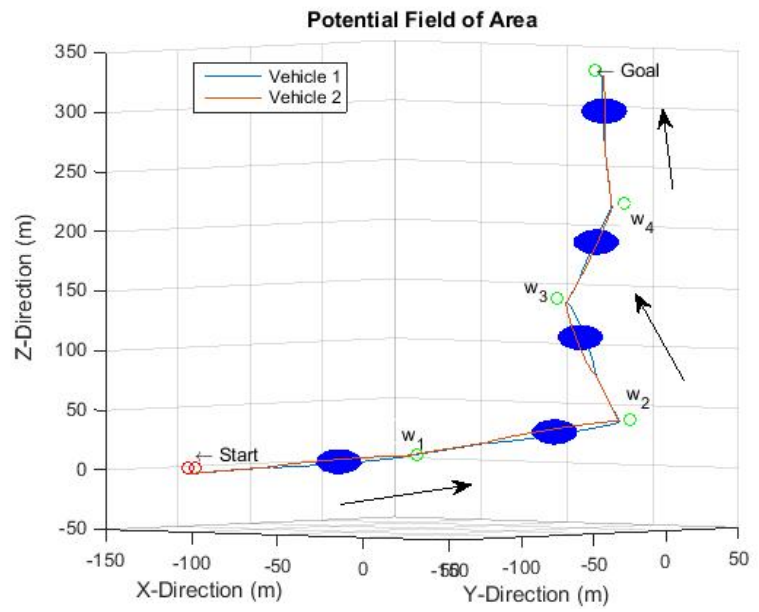

Fig. 4. The Survey of Five Objects using 2D Sub-Planes

A measure of the inter-vehicle distance was recorded at each iteration of the program using both techniques. A comparison of these results can be seen in Fig 5.

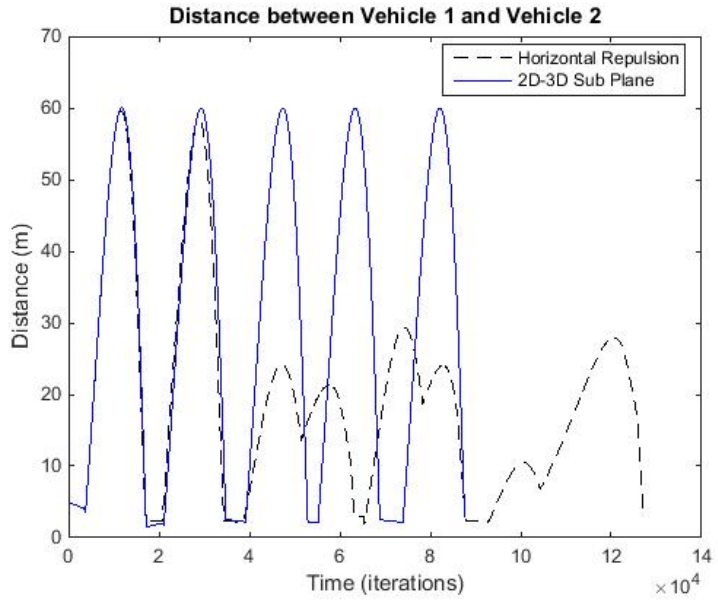

Fig. 5. Inter-Vehicle Distance Using Both Techniques 
Figures 3 and 5 show that using horizontal repulsion within a 3D environment can be effective, provided vehicles approach each object in a horizontal direction. However, as the vertical trajectory increases it can be clearly seen that not only do $v_{1} \&$ $v_{2}$ fail to produce a smooth trajectory (Fig 3) but their journey is $40 \%$ longer in duration (Fig 5).

Fig 4 shows both vehicles encircling each object smoothly, irrespective of the vertical incline. To validate this numerically, each object has a radius of $10 \mathrm{~m}$ coupled with a range of repulsion $20 \mathrm{~m}$ from its surface, therefore, with an ideal trajectory using opposing rotational fields, it would be expected that the maximum inter-vehicle separation around each object would be $60 \mathrm{~m}$. Table II displays the maximum distances between vehicles during object survey, using both techniques. Results obtained for the latter three objects (requiring a steeper approach) show that the average maximum distance, using horizontal repulsion, is $27.14 \mathrm{~m}-54.77 \%$ below the desired length. On the other hand, the proposed technique shows a drop of just $0.02 \%$, an average of $59.99 \mathrm{~m}$.

TABLE II

INTER-VEHICLE DISTANCE FOR BOTH HORIZONTAL REPULSION AND PROPOSED APPROACH

\begin{tabular}{|l|l|l|l|l|l|}
\hline \multicolumn{5}{|c|}{ Maximum distance between vehicles during object survey (m) } \\
\hline & Object 1 & Object 2 & Object 3 & Object 4 & Object 5 \\
\hline Horizontal & 59.58 & 58.47 & 24.18 & 29.32 & 27.93 \\
\hline 2D-3D & 60.01 & 59.99 & 59.99 & 60.00 & 59.99 \\
\hline
\end{tabular}

\section{CONCLUSION}

A new technique was proposed for the mapping/survey of underwater objects by multiple vehicles using traditional potential fields (APFs) alongside rotational potential fields (RPFs) within a 3D environment. Vehicles were maintained in a fluid formation when moving towards objects, then split in order to survey each object on opposite sides of its boundary utilising RPFs within a 2D sub plane. Simulations were carried out on a scenario using two vehicles to survey five objects. The results show the technique to provide much greater trajectory stability with a reduced journey time, compared to RPFs operating in a single direction.

\section{ACKNOWLEDGMENT}

The first author would like to acknowledge the Department of Employment and Learning, Northern Ireland for funding the research.

\section{REFERENCES}

[1] SMD. (2016) Product home page. [Online]. Available: https://smd.co.uk/products/trenchers-self-propelled/qtrencher-2800.htm

[2] O. ROV. (2016) Product home page. [Online]. Available: http://www.openrov.com/

[3] S. Pai and R. Hine, "Successful execution of remotely piloted autonomous marine vehicles to conduct METOC and Turbidity surveys," in 2014 IEEE/OES Autonomous Underwater Vehicles (AUV). Oxford, England: IEEE, October 2014, pp. 1-3.

[4] D. Bingham, T. Drake, U. Kingdom, and A. Hill, "The Application of Autonomous Underwater Vehicle (AUV) Technology in the Oil Industry Vision and Experiences," TS4.4 Hydrographic Surveying II, vol. XXII, pp. 1-13, 2002.

[5] T. Salgado-Jimenez, J. L. Gonzalez-Lopez, J. C. Pedraza-Ortega, L. G. Garcia-Valdovinos, L. F. Martinez-Soto, and P. A. Resendiz-Gonzalez, "Design of ROVs for the Mexican power and oil industries." Montreal, Canada: IEEE, October 2010, pp. 1-8.
[6] B. Fletcher, "UUV master plan: a vision for navy UUV development," in OCEANS 2000 MTS/IEEE Conference and Exhibition. Conference Proceedings (Cat. No.00CH37158), vol. 1. Providence, RI: IEEE, September 2000, pp. 65-71.

[7] M. Federis. (2016, July) Growing popularity of underwater drones raises similar concerns as aerial counterparts. [Online]. Available: http://www.capradio.org/articles/2016/07/01/growing-popularityof-underwater-drones-raises-new-questions/

[8] T. O. P. Team. (2016, October) Gulf oil spill. [Online]. Available: http://ocean.si.edu/gulf-oil-spill

[9] S. Evans. (2016, January) Mh370 search drone disappears after slamming into underwater mud volcano during missing plane hunt. [Online]. Available: http://www.mirror.co.uk/news/uknews/mh370-search-drone-disappears-after-7239581

[10] M. Dias, R. Zlot, N. Kalra, and A. Stentz, "Market-Based Multirobot Coordination: A Survey and Analysis," Proceedings of the IEEE, vol. 94, no. 7, pp. 1257-1270, July 2006.

[11] X. Xiang, B. Jouvencel, and O. Parodi, "Coordinated Formation Control of Multiple Autonomous Underwater Vehicles for Pipeline Inspection," International Journal of Advanced Robotic Systems, vol. 7, no. 1, pp. 75-84, March 2010.

[12] A. Sehgal, D. Cernea, and A. Birk, "Modeling underwater acoustic communications for multi-robot missions in a robotics simulator," in Oceans'10 IEEE Sydney. Australia: IEEE, May 2010, pp. 1-6.

[13] J. Mccolgan and E. W. Mcgookin, "Coordination of a School of Robotic Fish using Nearest Neighbour Principles," in OCEANS 2014 - TAIPEI. Taipei: IEEE, April 2014, pp. 1-8.

[14] D. Edwards, T. Bean, D. Odell, and M. Anderson, "A Leader-Follower Algorithm for Multiple AUV Formations," 2004 IEEE/OES Autonomous Underwater Vehicles (IEEE Cat. No.04CH37578), pp. 40-46, June 2004.

[15] C. B. Low, "A Dynamic Virtual Structure Formation Control for Fixed-Wing UAVs," in IEEE International Conference on Control and Automation, ICCA, Santiago, Chile, December 2011, pp. 627-632.

[16] O. Khatib, "Real-Time Obstacle Avoidance for Manipulators and Mobile Robots," in Robotics and Automation. Proceedings. 1985 IEEE International Conference on (Volume:2 ), March 1985, pp. 500-505.

[17] J. M. Esposito, "Decentralized cooperative manipulation with a swarm of mobile robots," 2009 IEEE/RSJ International Conference on Intelligent Robots and Systems, IROS 2009, pp. 5333-5338, June - July 2009.

[18] X. Chen and J. Zhang, "The three-dimension path planning of uav based on improved artificial potential field in dynamic environment," in Intelligent Human-Machine Systems and Cybernetics (IHMSC), 2013 5th International Conference on, vol. 2. IEEE, 2013, pp. 144-147.

[19] B. K. Sahu, B. Subudhi, and B. K. Dash, "Flocking control of multiple autonomous underwater vehicles," in 2012 Annual IEEE India Conference (INDICON). IEEE, 2012, pp. 257-262.

[20] Q. Zhu, Y. Yan, and Z. Xing, "Robot Path Planning Based on Artificial Potential Field Approach with Simulated Annealing," in Sixth International Conference on Intelligent Systems Design and Applications, vol. 2, Jinan, October 2006, pp. 622-627.

[21] S. Molotchnikoff and J. Rouat, Visual Cortex - Current Status and Perspectives. The Authors, 2012.

[22] J. Sfeir, M. Saad, and H. Saliah-Hassane, "An improved artificial potential field approach to real-time mobile robot path planning in an unknown environment," in Robotic and Sensors Environments (ROSE), 2011 IEEE International Symposium on. IEEE, 2011, pp. 208-213.

[23] A. D. Dang and J. Horn, "Path Planning for a Formation of Autonomous Robots in an Unknown Environment Using Artificial Force Fields," Proceedings of the 18th International Conference on System Theory, Control and Computing, Sinaia, Romania, October 17-19, 2014, pp. 773-777, October 2014.

[24] H. Rezaee, S. Member, and F. Abdollahi, "Mobile Robots Cooperative Control and Obstacle Avoidance Using Potential Field," Proceedings of the 2011 IEEE/ASME International Conference on Advanced Mechatronics (AIM2011), July 2011.

[25] H. Rezaee and F. Abdollahi, "Adaptive artificial potential field approach for obstacle avoidance of unmanned aircrafts," in 2012 IEEE/ASME International Conference on Advanced Intelligent Mechatronics (AIM). IEEE, 2012, pp. 1-6.

[26] D. McIntyre, W. Naeem, and C. Zhang, "Cooperative mapping and exploration using counter-rotational potential fields," in Signals and Systems Conference (ISSC), 2016 27th Irish. IEEE, 2016, pp. 1-7. 